\title{
Field-Theoretical Investigations of the Influence of Mutual Coupling Effects on the Capacity of MIMO Wireless Links
}

\author{
H. Ndoumbè Mbonjo Mbonjo, G. Wu, and V. Hansen \\ Chair of Electromagnetic Theory, University Wuppertal, Rainer-Gruenter-Str. 21, 42119 Wuppertal, Germany
}

\begin{abstract}
We present a MIMO channel model which takes into account mutual coupling effects at the receiver and transmitter array in order to assess the influence of mutual coupling effects on the capacity of MIMO channels. We evaluate the mutual impedances using a general approach based on the electric field integral equation (EFIE) and its implementation by the method of moments (MOM). We compute the capacity of a $2 \times 2-\mathrm{MIMO}$ system in a one path scenario for square half wavelength patch antenna elements and half wavelength dipole antenna elements. The capacity of the MIMO system with and without coupling increases compared to the single antenna transmission for the patch antenna elements. On the contrary for half wavelength dipole antenna elements we have found that the MIMO system degenerates to a one-transmitting, one-receiving antenna system due to mutual coupling.
\end{abstract}

\section{Introduction}

In Multiple Input Multiple Output (MIMO) systems antenna arrays are used at both the receiver ( $M$ antenna elements) and transmitter ( $N$ antenna elements) side. It was shown in (Telatar, 1999; Foschini and Gans, 1998) that MIMO systems improve potentially the capacity of wireless links and that under certain circumstances the capacity gain of a MIMO system scales with the number of transmitting and receiving antennas. But in these assumptions mutual coupling effects between antenna elements are ignored. However, mutual coupling is an important electromagnetic characteristic of an antenna array and therefore can considerably affect its performance. Especially, due to mutual coupling the currents on the antenna elements of an array interact. This effect is characterized by the mutual impedance and becomes meaningful for closely spaced antenna elements. Thus it is necessary to

Correspondence to: $\mathrm{H}$. Ndoumbè Mbonjo Mbonjo

(mbonjo@uni-wuppertal.de) include mutual coupling in the MIMO channel modelling. The influence of mutual coupling on MIMO systems was investigated in (Svantesson, 2001; Chiurtu, 2002) for arrays of dipole antennas starting with approximated analytical expressions for the mutual impedances between dipole antennas of finite length available in the common antenna literature (Balanis, 1982). However, other types of antenna elements, e.g. patch array antennas, can also be employed in MIMO systems. Thus it is reasonable to develop a general approach for the computation of the mutual impedance.

This paper is organized as follows: In Sect. 2 the modelling of mutual coupling effects in MIMO channels is presented. The computation of the mutual impedance is outlined in Sect. 3. Section 4 contains numerical results obtained from our computations. In Sect. 5 the conclusion of this work is presented.

\section{Modelling of mutual coupling in MIMO channel}

The starting point of our investigation is the voltage transfer matrix of a MIMO system as given in (Chiurtu, 2002). The MIMO voltage transfer matrix is obtained from the impedance matrix representation

$$
\left(\begin{array}{l}
\boldsymbol{U}_{s} \\
\boldsymbol{U}_{r}
\end{array}\right)=\left(\begin{array}{ll}
\mathbf{A} & \mathbf{B} \\
\mathbf{C} & \mathbf{D}
\end{array}\right)\left(\begin{array}{l}
\boldsymbol{I}_{s} \\
\boldsymbol{I}_{r}
\end{array}\right)
$$

of a $M+N$-port model of a MIMO system using $M$ transmitting antenna elements and $N$ receiving antenna elements where each antenna is associated with a port $i=1 . .(M+N)$. This model is depicted in Fig. 1. The ports associated with the transmitter array are on the left and those related to the receiver array are on the right. In Eq. (1) $\boldsymbol{U}_{r}$ resp. $\boldsymbol{I}_{r}$ are the column vectors of voltages resp. currents at the receiver and $\boldsymbol{U}_{s}$ resp. $\boldsymbol{I}_{s}$ denote the column vectors of the applied voltages resp. the input currents at the transmitter array.

- A is a $M \times M$ mutual impedance matrix of the transmitter array whose elements $A_{i j}, i=1 . . M, j=1 . . M$ 


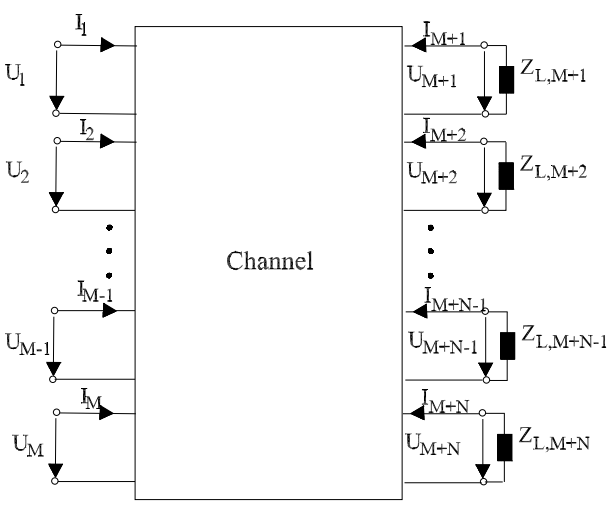

Fig. 1. Multi-port model of a MIMO system.

correspond to the mutual impedance between antenna element $i$ and antenna element $j$ of the transmitter array.

- B is a $M \times N$ MIMO channel transfer matrix whose elements $B_{i j}, i=1 . . M, j=1 . . N$ correspond to the channel transfer impedance between antenna element $i$ of the transmitter array and antenna element $j$ of the receiver array.

- C is a $N \times M$ MIMO channel transfer matrix whose elements $C_{i j}, i=1 . . N, j=1 . . M$ correspond to the channel transfer impedance between antenna element $i$ of the receiver array and antenna element $j$ of the transmitter array and

- D is a $N \times N$ mutual impedance matrix of the receiver array whose elements $D_{i j}, i=1 . . N, j=1 . . N$ represent the mutual impedance between antenna element $i$ and antenna element $j$ of the receiver array.

It follows from reciprocity that $\mathbf{C}$ is the transpose of $\mathbf{B}$ i.e. $\mathbf{C}=\mathbf{B}^{T}$. Assuming that each port $i=M+1 . .(M+N)$ of the receiver array is terminated by a load impedance $Z_{L, i}$ the currents $\boldsymbol{I}_{r}$ and voltages $\boldsymbol{U}_{r}$ at the receiver array are related through:

$\boldsymbol{U}_{r}=-\mathbf{Z}_{L} \boldsymbol{I}_{r}$

$\mathbf{Z}_{L}$ represents the diagonal matrix of the load impedances at the receiver array. Inserting Eq. (2) into Eq. (1) we solve for $\boldsymbol{U}_{r}=f\left(\boldsymbol{U}_{s}\right)$ and obtain

$\mathbf{T}=\left(\mathbf{I}_{N}+\mathbf{D Z}_{L}^{-1}-\mathbf{B}^{T} \mathbf{A}^{-1} \mathbf{B Z}_{L}^{-1}\right)^{-1} \mathbf{B}^{T} \mathbf{A}^{-1}$

for the voltage transfer matrix $\mathbf{T}$ of the MIMO system. In Eq. (3) $\mathbf{I}_{N}$ is the $(N \times N)$-identity matrix. Without mutual coupling $\mathbf{A}$ and $\mathbf{D}$ are diagonal matrices whose entries are the self impedances of the receiving resp. transmitting antenna elements.

\section{Computation of mutual impedance}

The mutual impedance between two antennas (denoted as antenna 1 and antenna 2) is derived from the reaction and reciprocity theorems (Unger, 1967; Balanis, 1982) and is given as

$Z_{12}=-\frac{1}{I_{1} I_{2}} \int_{r_{21}}^{r_{22}} \boldsymbol{E}_{12}\left(\boldsymbol{r}_{2}\right) \boldsymbol{J}_{2}\left(\boldsymbol{r}_{2}\right) d r_{2}$

where $\boldsymbol{J}_{2}\left(\boldsymbol{r}_{2}\right)$ is the current distribution on antenna 2 and $\boldsymbol{E}_{12}\left(\boldsymbol{r}_{2}\right)$ is the electric field produced by the current distribution $\boldsymbol{J}_{1}\left(\boldsymbol{r}_{1}\right)$ on antenna 1 along antenna 2. $I_{1}$ and $I_{2}$ are the input currents of the antennas. Using fundamental electromagnetic theory (Green's function concept) (Balanis, 1982) $\boldsymbol{E}_{12}\left(\boldsymbol{r}_{2}\right)$ is related to $\boldsymbol{J}_{1}\left(\boldsymbol{r}_{1}\right)$ by

$\boldsymbol{E}_{12}\left(\boldsymbol{r}_{2}\right)=\int_{r_{11}}^{r_{12}} \stackrel{\leftrightarrow}{G}_{J}^{E}\left(\boldsymbol{r}_{1}, \boldsymbol{r}_{2}\right) \boldsymbol{J}_{1}\left(\boldsymbol{r}_{1}\right) d r_{1}$.

The integrations in Eq. (4) resp. in Eq. (5) are performed over the entire regions of antenna 2 resp. antenna 1. In Eq. (5) $\stackrel{\leftrightarrow}{G}$ $\left(\boldsymbol{r}_{1}, \boldsymbol{r}_{2}\right)$ is the Green's function for the electric field. Inserting Eq. (5) into Eq. (4) we obtain

$Z_{12}=-\frac{1}{I_{1} I_{2}} \int_{r_{21}}^{r_{22}} \int_{r_{11}}^{r_{12}} \stackrel{\leftrightarrow}{G}_{J}^{E}\left(\boldsymbol{r}_{1}, \boldsymbol{r}_{2}\right) \boldsymbol{J}_{1}\left(\boldsymbol{r}_{1}\right) \boldsymbol{J}_{2}\left(\boldsymbol{r}_{2}\right) d r_{1} d r_{2}$

for the mutual impedance $Z_{12}$ between antenna 1 and 2 . Equations for the Green's function $\stackrel{\leftrightarrow}{G}_{J}^{E}\left(\boldsymbol{r}_{1}, \boldsymbol{r}_{2}\right)$ are available for several kinds of space (free space (Mittra, 1973), layered media (Hansen, 1988), half space as a special case of layered media). Only in some special cases where the current distribution on the antennas is approximately known (for example thin wire dipoles in free space) it is possible to give an analytically closed expression for the mutual impedance between the antennas. Therefore the current distribution on the antennas is evaluated numerically with the Method of Moments (MOM) (Harrington, 1968). In the MOM the antenna structure is divided into subregions and the unknown current distribution $\boldsymbol{J}(\boldsymbol{r})$ on the antenna is evaluated using a linear combination of $S_{n}$ basis functions $\boldsymbol{\beta}_{1, . ., S_{n}}(\boldsymbol{r})$ with unknown amplitudes $\hat{J}_{1 . . S_{n}}$ i.e.

$\boldsymbol{J}(\boldsymbol{r})=\sum_{n=1}^{S_{n}} \hat{J}_{n} \boldsymbol{\beta}_{n}(\boldsymbol{r})$

The unknown amplitudes of the current basis functions are solved using the electric field integral equation (EFIE) procedure: Eq. (7) is inserted into an integral representation of the electric field such as Eq. (5) and the electric field is evaluated at $S_{m}$ test points with simultaneous consideration of the boundary conditions for the electric field. Then the result is weighted with a function $\boldsymbol{\alpha}_{1 . . S_{m}}(\boldsymbol{r})$. Applying these three steps we obtain the matrix equation

$U_{n}=\tilde{Z}_{\left(m=1 . . S_{m}, n=1 . . S_{n}\right)} \hat{J}_{n}$. 

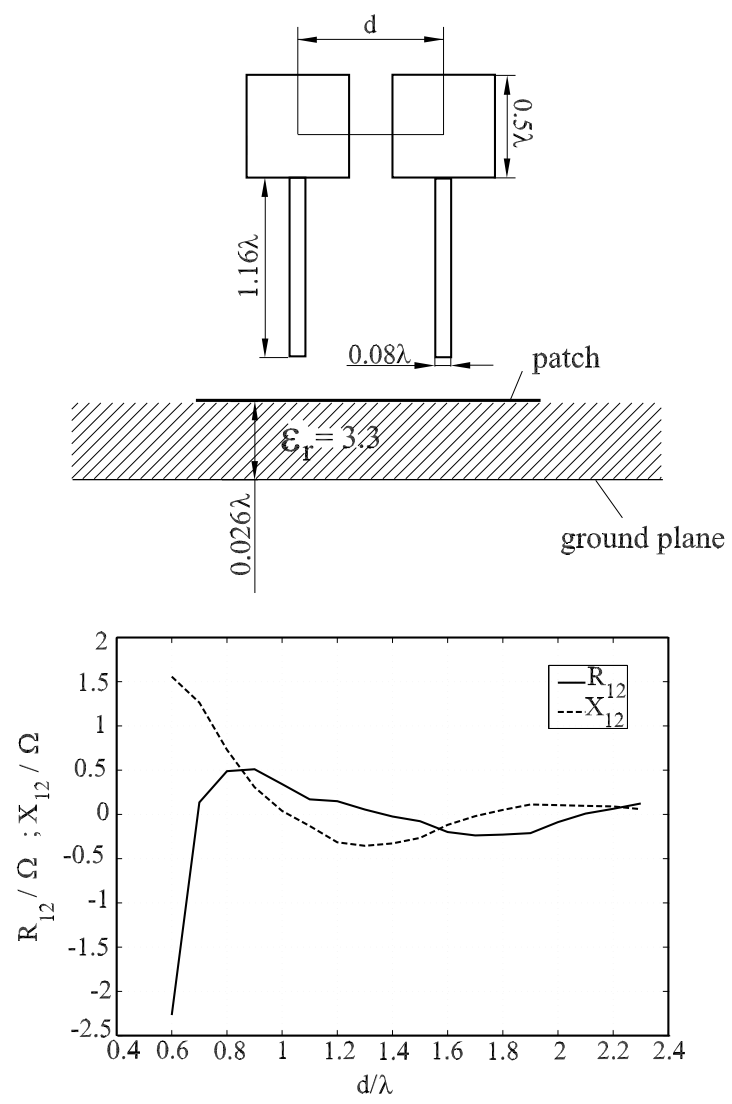

Fig. 2. Mutual impedance between two square half wavelength patch antennas as function of their separation.

Each element $\tilde{Z}_{m n}$ of the $\left(S_{m} \times S_{n}\right)-\tilde{Z}$ matrix represents the interaction between basis function $\boldsymbol{\beta}_{n}, n=1 . . S_{n}$ and test function $\boldsymbol{\alpha}_{m}, m=1 . . S_{m}$ and is given by

$\tilde{Z}_{m n}=\int_{r_{m 1}}^{r_{m 2}} \int_{r_{n 1}}^{r_{n 2}} \stackrel{\leftrightarrow}{G}_{J}^{E}\left(\boldsymbol{r}_{n}, \boldsymbol{r}_{m}\right) \boldsymbol{\beta}_{n}\left(\boldsymbol{r}_{n}\right) \boldsymbol{\alpha}_{m}\left(\boldsymbol{r}_{m}\right) d r_{n} d r_{m}$.

In Eq. (8) $U_{n}=\left(U_{1}, U_{2}, \ldots, U_{S_{n}}\right)^{T}$ denotes the voltage vector whose entries are zero except at the antenna feed point. Equation (8) is then solved for the unknown current coefficients $\hat{J}_{1 . . S_{n}}$.

From the MOM solution we obtain

$\boldsymbol{J}_{1}\left(\boldsymbol{r}_{1}\right)=\sum_{n=1}^{S_{n}} \hat{J}_{1 n} \boldsymbol{\beta}_{n}\left(\boldsymbol{r}_{1}\right) \quad \boldsymbol{J}_{2}\left(\boldsymbol{r}_{2}\right)=\sum_{m=1}^{S_{m}} \hat{J}_{2 m} \boldsymbol{\beta}_{m}\left(\boldsymbol{r}_{2}\right)$

for antenna 1 resp. antenna 2. We include Eq. (10) in Eq. (6) and obtain

$Z_{12}=-\frac{1}{I_{1} I_{2}} \sum_{n=1}^{S_{n}} \sum_{m=1}^{S_{m}} \hat{J}_{1 n} \hat{J}_{2 m} \tilde{Z}_{m n}$

for the mutual impedance between antenna 1 and antenna 2 . For the computation of the $\tilde{Z}_{m n}$ we choose the test functions according to the Galerkin method (Sadiku, 2001) i.e. $\boldsymbol{\alpha}_{m}(\boldsymbol{r})=\boldsymbol{\beta}_{m}(\boldsymbol{r})$.

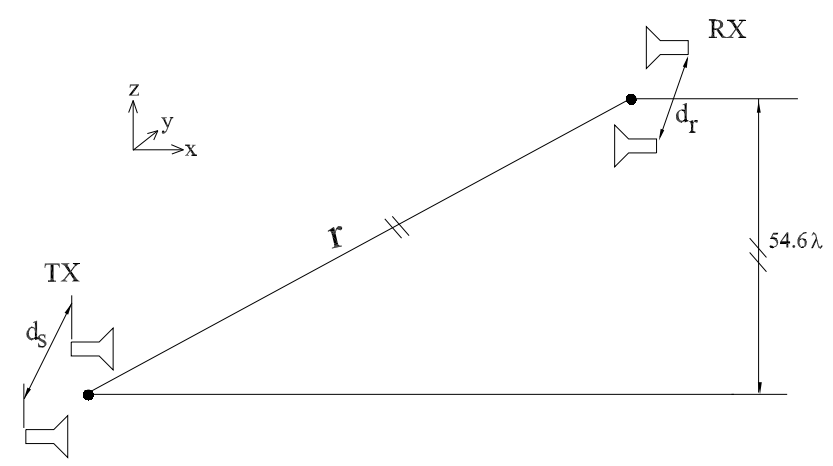

Fig. 3. One path scenario: the antennas are in free space.

Figure 2 depicts the obtained values for the mutual impedance $Z_{12}=R_{12}+j X_{12}$ between two square half wavelength patch antennas for different spacings between the antennas. The computation of the patch antennas is based on a spectral domain analysis of an EFIE formulation applying the appropriate Green's functions of layered media (Vaupel, 1999).

\section{Numerical results}

In this section we present and discuss our numerical results. We compute the channel capacity of a MIMO system with $M=2$ transmitting and $N=2$ receiving antennas with and without coupling in the one path scenario depicted on Fig. 3 .

The capacity of a $N \times M$-MIMO system with no channel state information (CSI) at the transmitter i.e. when the channel is unknown to the transmitter is given by the following equation (Telatar, 1999)

$C=\sum_{i=1}^{k} \log _{2}\left(1+\frac{\lambda_{i} S N R}{M}\right) \quad \mathrm{bps} / \mathrm{Hz}$

In Eq. (12) $\lambda_{1}, \lambda_{2} \ldots \lambda_{k}$ are the nonzero eigenvalues of the matrix

$$
\begin{array}{rlll}
\mathbf{W} & =\mathbf{T T}^{H} & \text { for } & N \leq M \\
& =\mathbf{T}^{H} \mathbf{T} & \text { for } & M \leq N
\end{array}
$$

$k=\min (\mathrm{M}, \mathrm{N})$ and $S N R$ is the average signal to noise ratio at each receiving antenna. The superscript $H$ in Eq. (13) denotes the Hermitian transpose of $\mathbf{T}$. Equation (12) represents the capacity of a MIMO system as the sum of capacities of $k$ parallel single input single output (SISO) subchannels. The eigenvalues $\lambda_{1}, \lambda_{2} \ldots \lambda_{k}$ of the matrix $\mathbf{W}$ are the power gains of the subchannels. Figure 4 shows the obtained capacity values versus the SNR with and without coupling for patch antenna elements $\left(d_{s}=d_{r}=0.6 \lambda, r \approx 215 \lambda\right.$ with $\lambda$ the wavelength). The dashed curve represents the capacity values of a one-transmitting, one-receiving antenna system $(N=M=1)$. We set $Z_{L, 1}=Z_{L, 2}=50 \Omega$ for the load impedances at the receiver array. It follows from Fig. 4 that in this case the capacity of the MIMO system with and without coupling is greater 


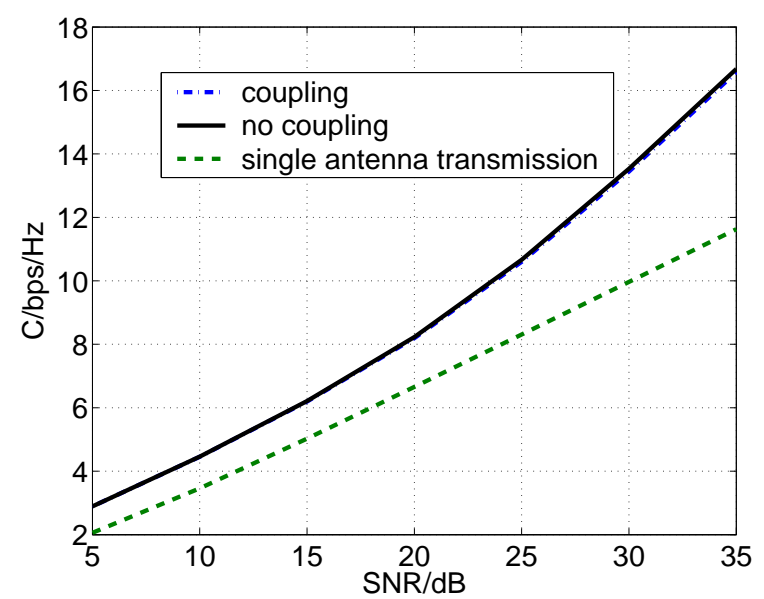

Fig. 4. Capacity of the MIMO system for patch antenna elements: $d_{s}=d_{r}=0.6 \lambda ; r \approx 215 \lambda$.

than the capacity of the SISO system. Furthermore there is no significant difference between the capacity of the MIMO system with and without coupling. This is due to the low coupling of the patch antennas.

Figure 5 shows the obtained capacity curve versus SNR with and without coupling for half wavelength dipoles ( $d_{s}=d_{r}=0.4 \lambda, r \approx 215 \lambda$ with $\lambda$ the wavelength). It follows from Fig. 5 that in this scenario the capacity of the MIMO system without consideration of mutual coupling increases compared to the capacity of a single antenna transmission system: We obtain approximately a $2 \mathrm{~dB}$ increase of the capacity for a $S N R$ value of $35 \mathrm{~dB}$. However, with mutual coupling the MIMO system degenerates in a one-transmitting, one-receiving antenna system and there is no capacity gain compared to the SISO case.

\section{Conclusions}

In this paper we have presented a MIMO channel model which considers mutual coupling effects at the receiver and transmitter arrays. We have obtained an expression for the MIMO voltage channel transfer matrix from the impedance matrix representation of the $M+N$ ( $M$ is the number of transmitting antennas elements and $N$ is the number of receiving antennas) multi-port model of a MIMO system. We have evaluated the mutual impedances using a general approach based on the electric field integral equation (EFIE) and its implementation by the method of moments (MOM). Further, we have computed the capacity of a $2 \times 2$-MIMO system ( $M=2$ transmitting antennas and $N=2$ receiving antennas) from our derived channel model with and without coupling in a one path scenario. We have used square patch antennas and half wavelength dipoles in a side-by-side configuration as transmitting and receiving antennas in our computations. The capacity of the MIMO system with and without coupling increases compared to the single antenna transmission for the patch antenna elements. On the contrary for

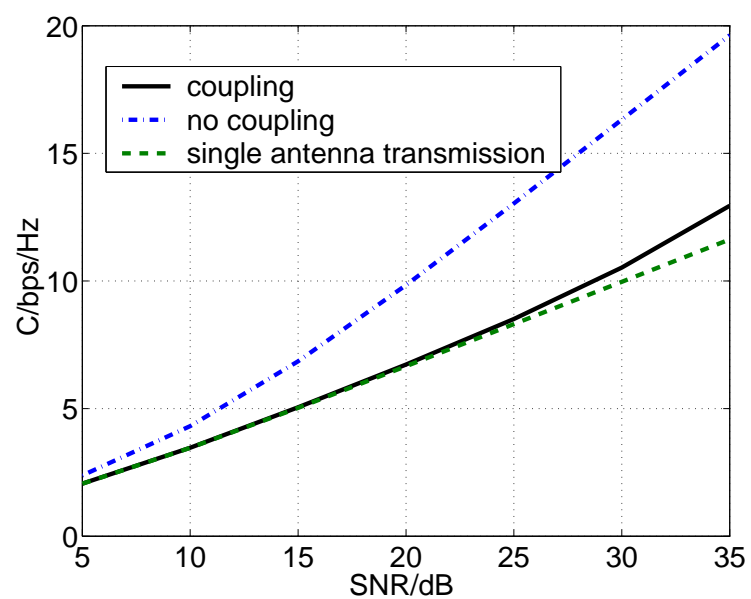

Fig. 5. Capacity of the MIMO system for half wavelength antenna elements: $d_{s}=d_{r}=0.4 \lambda ; r \approx 215 \lambda$.

half wavelength dipoles antenna elements we have found that the MIMO system degenerates in a one-transmitting, onereceiving antenna system due to mutual coupling.

The results presented in this paper demonstrate that mutual coupling can have a significant impact on MIMO systems. Therefore mutual coupling between antenna elements should be taken into account in the modelling of MIMO channels.

\section{References}

Telatar, I. E.: Capacity of Multi-Antenna Gaussian Channels, European Transactions on Communications, vol. 10, no. 6, 585-595, 1999.

Foschini, G. J. and Gans, M.: J. On Limits of Wireless Communications in a Fading Environment When Using Multiple Antennas, Wireless Personal Communications, vol. 6, 311-335, 1998.

Svantesson, T. and Ranheim, A.: Mutual Coupling Effects on the Capacity of Multielement Antenna Systems, IEEE International Conference on Acoustics, Speech, and Signal Processing, Salt Lake City, USA, May 2001.

Chiurtu, N.: Multiple Antenna Systems for Mobile Communications, $\mathrm{PhD}$ Thesis, Swiss Federal Institute of Technology (EPFL), Lausanne, Switzerland, December 2002.

Balanis, C.: Antenna Theory: Analysis and Design, John Wiley \& Sons, New York 1982.

Unger, H. G.: Elektromagnetische Wellen II, Vieweg \& Sohn Verlag, Braunschweig 1967.

Mittra, R.: Computer Techniques for Electromagnetics, Pergamon Press, Oxford 1973.

Hansen, V.: Numerical Solution of Antennas in Layered Media, John Wiley \& Sons, New York, 1988.

Harrington, R. F.: Field Computation by Moment Methods, Cazenovia, New York 1968.

Matthew Sadiku, N. O.: Numerical Techniques in Electromagnetics, CRC Press, Boca Raton, 2001.

Vaupel, T.: Elektrodynamische Analyse von (M)MICund Submm-Wellenstrukturen basierend auf einem Oberflächen/Volumenintegralgleichungsverfahren, $\mathrm{PhD}$ Thesis (in German), University of Wuppertal, Germany, January 1999. 